Grazielle Ferreira Iroldi

O https://orcid.org/0000-0002-6538-7280

Élen dos Santos Alves²

Ohttps://orcid.org/0000-0001-9696-2703

Bruna Moretti Luchesi ${ }^{3}$

@inttps://orcid.org/0000-0002-0508-0818

Juliana de Fátima Zacarin Cardoso ${ }^{4}$

O- https://orcid.org/0000-0001-5053-0868

Sofia Cristina lost Pavarini ${ }^{5}$

Ohttps://orcid.org/0000-0001-9359-8600

Keika Inouye

OChtps://orcid.org/0000-0003-3570-0704

\section{Associações entre estresse, sintomas depressivos e insônia em idosos}

\author{
Associations among stress, depressive symptoms, and insomnia in elders
}

DOI: 10.1590/0047-2085000000287

\section{RESUMO}

Objetivos: Identificar associação entre variáveis emocionais (estresse e sintomas depressivos) e o autorrelato de dificuldade para dormir em idosos. Métodos: Estudo descritivo, quantitativo e de corte transversal com 341 idosos cadastrados nas Unidades de Saúde da Família do município de São Carlos - SP, divididos em: (a) grupo sem dificuldade para dormir autorrelatada e (b) grupo com dificuldade para dormir autorrelatada. Os instrumentos para a coleta de dados foram: Ficha de Caracterização do Idoso com Questão Específica de Dificuldade para Dormir Autorrelatada, Escala de Estresse Percebido e Escala de Depressão Geriátrica. Resultados: A dificuldade para dormir foi autorrelatada em 47,5\% da amostra ( $n=162$ ). Os níveis de estresse no grupo de idosos com dificuldade para dormir mostrou-se mais elevado ( $M=21,29$ pontos) que no grupo sem dificuldade para dormir $(M=15,97)(U=10034,50$; $p=0,010)$. Os idosos com dificuldade para dormir apresentavam maior número de sintomas depressivos ( $M=4,41$ pontos) quando comparados aos sem dificuldade para dormir ( $M=3,09$ pontos) $(U=$ 10427,50, $p=0,000)$. Conclusão: $O$ estudo observou que idosos que autorrelataram dificuldade para dormir apresentaram níveis de estresse mais elevados e maiores escores na escala de sintomas depressivos, permitindo concluir que há associação entre variáveis emocionais e dificuldade para dormir em idosos.

\section{PALAVRAS-CHAVE}

Sono, estresse, depressão, idoso.

\section{ABSTRACT}

Objectives: To identify an association between emotional variables (stress and depressive symptoms) and the self-report of difficulty sleeping in the elderly. Methods: Descriptive, quantitative and cross-sectional study with 341 elderly people registered in Family Health Units in the city of São Carlos - SP were interviewed, divided into (a) Self-reported group without difficulty and (b) Group with difficulty sleeping self-reported. The instruments for data collection were Characterization Sheet for the Elderly with Specific Question of Difficulty in Self-Reported Sleep, Perceived Stress Scale and Geriatric Depression Scale. Results: The difficulty to sleep was self-reported in $47.5 \%$ of the sample $(n=162)$. The stress levels of the elderly group with difficulty sleeping were higher ( $M=21.29$ points) than in the group without difficulty sleeping $(M=15.97)(U=10034.50 ; p=0.010)$. In addition, elderly people with difficulty sleeping had a higher number of depressive symptoms ( $M=4.41$ points) when compared to those without difficulty sleeping $(M=3.09$ points) $(U=10427.50, p=0.000)$. Conclusion: The study observed that elderly people who self-reported difficulty sleeping had higher levels of stress and higher scores on the scale of depressive symptoms, allowing concluding that there is an association between emotional variables and difficulty sleeping in the elderly.

\section{KEYWORDS}

Sleep, stress, depression, elderly.

\footnotetext{
Received in: Apr/29/2020. Approved in: Aug/10/2020

1 Universidade Federal de São Carlos, Departamento de Gerontologia, São Carlos, SP, Brasil.

2 Universidade Federal de São Carlos, Departamento de Enfermagem, Programa de Pós-Graduação em Enfermagem, São Carlos, SP, Brasil.

3 Universidade Federal do Mato Grosso do Sul, Departamento de Medicina, Campos de Três Lagoas, Mato Grosso do Sul, MS, Brasil.

4 Universidade Federal de São Carlos, Programa de Pós-Graduação em Enfermagem, São Carlos, SP, Brasil.

5 Universidade Federal de São Carlos, Departamento de Gerontologia, Programa de Pós-Graduação em Enfermagem e Programa de Pós-Graduação em Gerontologia, São Carlos, SP, Brasil.

6 Universidade Federal de São Carlos, Departamento de Gerontologia e Programa de Pós-Graduação em Gerontologia, São Carlos, SP, Brasil.
}

Address for correspondence: Grazielle Ferreira Iroldi. Rodovia Washington Luiz, s/n - 13565-905 - São Carlos, SP, Brasil. E-mail: graziiroldi@gmail.com 


\section{INTRODUÇÃO}

As doenças crônicas e seus agravos são fatores que aumentam o risco de comprometimento funcional e emocional em idosos. Uma pesquisa realizada pela Organização Pan-Americana de Saúde (Opas) destacou estresse e sintomas depressivos entre os agravos que afetam os idosos'. Além disso, alterações e distúrbios do sono relacionados com o envelhecimento são frequentemente documentados na literatura ${ }^{2-5}$.

O estudo de Müller e Guimarães ${ }^{6}$ aponta que os distúrbios do sono, especialmente a insônia, estão associados a transtornos psiquiátricos como sintomas depressivos. Lucchesi et al. ${ }^{7}$ citaram que cerca de $80 \%$ dos pacientes com sintomas depressivos queixam-se de mudanças nos padrões do sono. Além disso, pacientes com transtornos ansiosos apresentam frequentes queixas sobre dificuldade para relaxar ou parar de se preocupar com seus problemas quando estão no leito.

Ferreira ${ }^{8}$, no que se refere aos aspectos emocionais, destacou que a dificuldade em dormir pode estar diretamente correlacionada ao desenvolvimento de psicopatologias. A autora advertiu que a frequente dificuldade de dormir e a insônia têm impacto na prevalência de humor deprimido e ansiedade.

O sono é uma necessidade humana fundamental que influencia no bem-estar e na qualidade de vida do indivíduo'. Entre os idosos, observam-se frequentemente queixas relacionadas à dificuldade para dormir, aumento do período para o início do sono, despertares noturnos, sonolência diurna, insônia, dificuldades respiratórias e ronco ${ }^{3,4}$.

Ao envelhecer, o sono torna-se progressivamente fragmentado e com interrupções, resultando em diminuição de sua eficiência, sonolência ao longo do dia, diminuição do estado de alerta e necessidade de cochilos curtos, ocasionando a diminuição da quantidade total de horas do sono no período de 24 horas $^{5}$.

O Manual Diagnóstico e Estatístico de Transtornos Mentais (DSM-5) define o transtorno depressivo maior como uma condição clássica que representa um grupo de transtornos caracterizados por episódios distintos, de pelo menos duas semanas de duração, que envolvem alterações de afeto, cognição e funções neurovegetativas. Algumas características comuns desses transtornos são humor triste, vazio ou irritável, acompanhado de alterações físicas e cognitivas que afetam significativamente a capacidade de funcionamento dos indivíduos ${ }^{10,11}$.

Ferreira et al. ${ }^{12}$, em um estudo analítico entre as relações de experiência de eventos estressantes associados ao cuidado, a sintomas depressivos e a distúrbios do sono em homens e mulheres idosos concluiu que a prevalência total de sintomas depressivos nos entrevistados foi de 33\%; quando analisado apenas o grupo das mulheres, esse número cresce para $36,5 \%$. Em relação ao sono, 37,7\% dos entrevistados pontuaram para distúrbios de sono. Além disso, o estudo também apresentou que idosos com sintomas depressivos apresentam maior risco para alterações de sono.

Hoffmann et al. ${ }^{13}$ apontaram que sintomas depressivos, doenças físicas, perda da independência e suicídio são muito mais comuns na velhice. A atenção a essas variáveis pode minimizar o risco de alterações cognitivas e de mortalidade entre idosos e reduzir gastos com cuidados e as questões de sobrecarga da família. Cole e Dendukuri' ${ }^{14}$, que fizeram uma metanálise de 20 estudos longitudinais publicados em francês e inglês, mostraram a importância de fatores de risco para sintomas depressivos entre idosos, como luto, distúrbios do sono, incapacidade, depressão anterior e sexo feminino.

Os estudos de Menezes-Silva et al..$^{15}$ e Xavier et al. ${ }^{16}$ mostraram que a ansiedade nos idosos pode estar relacionada às limitações vivenciadas na velhice. A Sociedade Brasileira de Sono ${ }^{17}$ ainda traz que a dificuldade para dormir também tem sido citada como prevalente na sociedade, entretanto os sintomas depressivos e ansiosos dificultam o diagnóstico e a determinação de causa e efeito. Sendo assim, torna-se necessária, durante o tratamento malsucedido do paciente com dificuldade para dormir, a verificação de sintomas depressivos e/ou da presença de transtorno de ansiedade generalizada. Os pacientes com insônia crônica, tais como os deprimidos, também apresentam uma maior incidência de queixas médicas, procurando, assim, mais serviços médicos que a população em geral, tendo uma qualidade de vida reduzida.

Uma revisão de literatura realizada por Chellappa e Araújo ${ }^{18}$, que teve como objetivo analisar as publicações sobre sono e suas alterações nos sintomas depressivos, além de modelos teóricos propostos para explicar essas alterações, analisou 20 artigos originais e 10 de revisão publicados entre 1991 e 2006. Os autores concluíram que os estudos têm apontado associação entre transtornos do sono e sintomas depressivos. Porém, os mecanismos fisiopatológicos dos distúrbios do sono nos sintomas depressivos mostram-se complexos e de natureza multifacetada.

Além disso, a prevalência de sintomas depressivos e queixas relacionadas ao sono, embora tenha sido bem discutida pela comunidade científica, especialmente em estudos internacionais, comtempla idosos de serviços especializados ou com perfil demográfico diferente da realidade brasileira ${ }^{14}$. Os idosos atendidos na atenção básica têm renda e escolaridade inferiores. Por isso, há a necessidade de estudos que contemplem a atenção primária para subsidiar e fortalecer estratégias e políticas de saúde em todos os níveis de atenção.

Dessa forma, o presente estudo tem como objetivo investigar a associação entre variáveis emocionais (estresse e sintomas depressivos) e dificuldade para dormir autorrelatada em idosos. Este artigo tem como hipótese de pesquisa que idosos com dificuldade para dormir têm maior número 
de sintomas depressivos e níveis de estresse mais elevados do que idosos sem dificuldade para dormir.

\section{MÉTODOS}

\section{Desenho do estudo, população e ética}

Tratou-se de um estudo descritivo, quantitativo e de corte transversal, realizado na cidade de São Carlos, SP. O número total de habitantes de São Carlos, em 2010, era de 221.692, e 12,91\% do total tinham 60 anos ou mais. Em 2020, a população passou para 242.632 e 16,85\% dela era de idosos ${ }^{19,20}$.

A coleta de dados foi realizada em 2014. Na época, o município contava com 16 Unidades de Saúde da Família (USFs). Atualmente, a cidade dispõe de 21 USFs, sendo duas localizadas em área rural. A cobertura das unidades no município é de 39.768 habitantes, correspondendo a uma cobertura de área de abrangência de 17,9\% do total da população ${ }^{21}$. A partir do número total aproximado de idosos atendidos nas USFs do município de São Carlos - 5.130 -, calculou-se que 341 idosos constituiriam uma amostra com nível de confiança de 95\% e margem de erro de 5,15\% (cálculo realizado por meio da plataforma Survey Monkeyं, disponível em https:// pt.surveymonkey.com/mp/sample-size-calculator/\#, acesso em julho de 2020).

Foram convidados a participar deste estudo idosos com mais de 60 anos de idade, cadastrados nas áreas de abrangências de todas as 16 USFs existentes na época da coleta no município. Foram excluídos idosos com distúrbios auditivos, visuais e/ou de linguagem que impedissem a aplicação dos instrumentos.

O procedimento de coleta de dados, bem como todas as etapas deste trabalho, seguiu rigorosamente os preceitos éticos conforme estabelecido na Resolução n 466/2012 do Conselho Nacional de Saúde ${ }^{22}$. O projeto foi aprovado pelo Comitê de Ética em Pesquisa, sendo o número de aprovação 711.592. Todos os participantes foram informados dos objetivos do trabalho, consultados quanto ao aceite em participar do estudo e assegurados do sigilo das informações individuais. Além disso, os indivíduos somente participaram da pesquisa após assinar o termo consentimento, no qual foi assegurado ao participante, a qualquer momento durante a coleta de dados, o direito de interromper sua participação sem compromisso de justificativa.

\section{Procedimento geral}

Primeiramente foi agendada uma visita às USFs para solicitar uma lista dos idosos cadastrados em suas respectivas áreas. A listagem inicial foi composta por 594 idosos. Os idosos foram contatados nos domicílios para verificar os critérios de inclusão e exclusão. Desses, 26 idosos haviam falecido, 84 se recusaram a participar da pesquisa e 105 mudaram de en- dereço ou não foram encontrados após três tentativas de visitas. Os idosos foram visitados e, quando preenchidos os critérios de elegibilidade, foram convidados a participar da pesquisa. Após o aceite, foram coletadas informações de caracterização sociodemográfica. Durante a coleta de dados, 38 idosos não completaram os instrumentos, havendo a perda, resultando em uma amostra final de 341 idosos.

Ao término da primeira etapa, foi agendado um horário para a coleta dos instrumentos: (a) Ficha de Caracterização do Idoso com Questão Específica de Dificuldade para Dormir Autorrelatada "O Sr.(a) tem dificuldade para dormir?" (com as opções de resposta "sim" ou "não"), elaborada pelos pesquisadores; (b) Escala de Estresse Percebido, elaborada por Cohen, Kamarck e Mermelstein (1983) ${ }^{23}$, validada e traduzida para o contexto brasileiro por Luft et al. (2007) ${ }^{24}$; a escala compreende 14 perguntas que aferem o grau de estresse percebido pelo idoso, com uma pontuação final que varia de zero a 56 pontos; quanto mais alta a pontuação, maior o grau de estresse percebido pelo idoso; e (c) Escala de Depressão Geriátrica (GDS-15), elaborada por Yesavage et al. (1983)25; sua versão original compreendia 30 itens; em 1986 os autores elaboraram uma versão reduzida com 15 itens; ambas as versões permitem o rastreio de sintomas depressivos em idosos; em 1999, Almeida e Almeida ${ }^{26}$ validaram ambas as versões; o escore final da escala pode variar de zero a 15 pontos, e quanto maior a pontuação, maiores são os indícios de sintomas depressivos. A coleta de dados foi realizada por pesquisadores treinados. Foram realizadas entrevistas no domicílio dos idosos ou em locais previamente combinados segundo a conveniência dos participantes.

\section{Procedimento de análise dos dados}

Os dados obtidos foram digitados em um banco no programa Statistical Package for Social Sciences (SPSS) para Windows, versão 19.0. Não foi verificada a normalidade dos dados pelo teste Kolmogorov-Smirnov ( $p=0,000$ ). Dessa forma, foram realizadas análises não paramétricas: descritivas para caracterizar o perfil dos idosos; teste de qui-quadrado de Pearson, teste de Mann-Whitney e teste Exato de Fischer.

\section{RESULTADOS}

\section{Caracterização sociodemográfica da amostra}

Neste estudo, foram avaliados 341 idosos, sendo eles divididos em dois grupos: o primeiro grupo, de idosos sem dificuldade para dormir autorrelatada $(52,5 \%, n=179)$; e o segundo grupo, de idosos com dificuldade para dormir autorrelatada $(47,5 \%, n=162)$.

Dentre os resultados, destaca-se que os indivíduos da amostra eram, em sua maioria, do sexo feminino ( $n=262 ; 76,8 \%)$, brancos $(n=238,69,8 \%)$, casados ou com 
companheiro ( $n=308 ; 90,3 \%$ ) e com baixa escolaridade 277 idosos $(81,2 \%)$ tinham no máximo o primário completo, que corresponde, atualmente, ao quarto ano do ensino fundamental. Em anos, a média foi de 3,81 anos de estudo $\left(\mathrm{Md}=4,00, \mathrm{DP}=3,52, \mathrm{X}_{\text {min }}=0,00, \mathrm{X}_{\text {máx }}=19,00\right)$. Em relação à religião, $64,5 \%$ eram católicos $(n=220)$ e $23,8 \%$ eram evangélicos $(n=81)$, sendo $76,8 \%(n=262)$ praticantes (Tabelas 1 e 2). A média de idade foi de 69,61 anos ( $M d=69,00, D P=$ $\left.7,12, X_{\min }=60,00, X_{\text {máx }}=98,00\right)$ e a da renda familiar foi, em reais, de $R \$ 2.302,21$ (Tabela 2).

A distribuição da amostra nos grupos segundo as características sociodemográficas sexo, situação conjugal, escolaridade, religião (praticante ou não) e etnia são apresentadas na tabela 1. A tabela 2 apresenta dados descritivos detalhados de idade, escolaridade (em anos) e renda familiar por grupos e na totalidade da amostra, bem como análises comparativas.

Tabela 1. Distribuição da amostra nos grupos (com e sem dificuldade para dormir) segundo as variáveis de caracterização e análise comparativa entre os grupos - São Carlos, 2014

\begin{tabular}{|c|c|c|c|c|c|c|c|c|c|}
\hline \multirow{2}{*}{$\begin{array}{l}\text { Grupos } \\
\text { Variáveis }\end{array}$} & \multicolumn{2}{|c|}{$\begin{array}{c}\text { Sem dificuldade para } \\
\text { dormir }\end{array}$} & \multicolumn{2}{|c|}{$\begin{array}{l}\text { Com dificuldade para } \\
\text { dormir }\end{array}$} & \multicolumn{2}{|c|}{ Todos os grupos } & \multicolumn{3}{|c|}{$\begin{array}{l}\text { Análise comparativa } \\
\text { Qui-quadrado de Pearson* } \\
\text { Teste exato de Fisher** }\end{array}$} \\
\hline & $\mathbf{N}$ & $\%$ & $\mathbf{N}$ & $\%$ & $\mathbf{N}$ & $\%$ & Valor & gl & p \\
\hline Sexo & & & & & & & $7,326^{\star}$ & 1 & $0,007^{\star \star \star}$ \\
\hline Feminino & 127 & 70,9 & 135 & 83,3 & 262 & 76,8 & & & \\
\hline Masculino & 52 & 29,1 & 27 & 16,7 & 79 & 23,2 & & & \\
\hline Total & 179 & 100 & 162 & 100 & 341 & 100 & & & \\
\hline Situação conjugal & & & & & & & $1,261^{\star *}$ & & 0,779 \\
\hline Casado(a) & 164 & 91,6 & 144 & 88,9 & 308 & 90,3 & & & \\
\hline Solteiro(a) & 7 & 3,9 & 7 & 4,3 & 14 & 4,1 & & & \\
\hline Divorciado(a) & 3 & 1,7 & 3 & 1,9 & 6 & 1,8 & & & \\
\hline Viúvo(a) & 5 & 2,8 & 8 & 4,9 & 13 & 3,8 & & & \\
\hline Total & 179 & 100 & 162 & 100 & 341 & 100 & & & \\
\hline Escolaridade & & & & & & & $5,787^{\star \star}$ & & 0,450 \\
\hline Nunca foi à escola & 36 & 20,1 & 38 & 23,5 & 74 & 21,7 & & & \\
\hline Curso alfabetizante & 5 & 2,8 & 5 & 3,1 & 10 & 2,9 & & & \\
\hline Primário & 99 & 55,3 & 94 & 58,0 & 193 & 56,6 & & & \\
\hline Ginásio & 21 & 11,7 & 10 & 6,2 & 31 & 9,1 & & & \\
\hline Científico/clássico & 10 & 5,6 & 12 & 7,4 & 22 & 6,5 & & & \\
\hline Curso superior & 6 & 3,4 & 2 & 1,2 & 8 & 2,3 & & & \\
\hline Pós-graduação & 2 & 1,1 & 1 & 0,6 & 3 & 0,9 & & & \\
\hline Total & 179 & 100 & 162 & 100 & 341 & 100 & & & \\
\hline Religião & & & & & & & $13,588^{* *}$ & & $0,048^{\star \star \star}$ \\
\hline Católico(a) & 120 & 67,0 & 100 & 61,7 & 220 & 64,5 & & & \\
\hline Evangélico(a) & 40 & 22,3 & 41 & 25,3 & 81 & 23,8 & & & \\
\hline Congregação cristã & 9 & 5,0 & 5 & 3,1 & 14 & 4,1 & & & \\
\hline Adventista & 2 & 1,1 & 3 & 1,9 & 5 & 1,5 & & & \\
\hline Espírita & 1 & 0,6 & 9 & 5,6 & 10 & 2,9 & & & \\
\hline Protestante & 0 & 0,0 & 1 & 0,6 & 1 & 0,3 & & & \\
\hline Budista & 1 & 0,6 & 0 & 0,0 & 1 & 0,3 & & & \\
\hline Umbanda & 0 & 0,0 & 1 & 0,6 & 1 & 0,3 & & & \\
\hline Não possui & 6 & 3,4 & 2 & 1,2 & 8 & 2,3 & & & \\
\hline Total & 179 & 100 & 162 & 100 & 341 & 100 & & & \\
\hline Praticante & & & & & & & $2,752^{*}$ & 2 & 0,253 \\
\hline Não & 40 & 22,3 & 36 & 22,2 & 76 & 22,3 & & & \\
\hline Sim & 136 & 76,0 & 126 & 77,8 & 262 & 76,8 & & & \\
\hline NR & 3 & 1,7 & 0 & 0,0 & 3 & 0,9 & & & \\
\hline Total & 179 & 100 & 162 & 100 & 341 & 100 & & & \\
\hline
\end{tabular}




\begin{tabular}{|c|c|c|c|c|c|c|c|c|c|}
\hline \multirow{2}{*}{$\begin{array}{l}\text { Grupos } \\
\text { Variáveis }\end{array}$} & \multicolumn{2}{|c|}{$\begin{array}{l}\text { Sem dificuldade para } \\
\text { dormir }\end{array}$} & \multicolumn{2}{|c|}{$\begin{array}{c}\text { Com dificuldade para } \\
\text { dormir }\end{array}$} & \multicolumn{2}{|c|}{ Todos os grupos } & \multicolumn{3}{|c|}{$\begin{array}{l}\text { Análise comparativa } \\
\text { Qui-quadrado de Pearson* } \\
\text { Teste exato de Fisher** }\end{array}$} \\
\hline & $\mathbf{N}$ & $\%$ & $\mathbf{N}$ & $\%$ & $\mathbf{N}$ & $\%$ & Valor & gl & p \\
\hline Etnia & & & & & & & $6,255^{\star *}$ & & 0,129 \\
\hline Branca & 127 & 70,9 & 111 & 68,5 & 238 & 69,8 & & & \\
\hline Preta & 9 & 5,0 & 19 & 11,7 & 28 & 8,2 & & & \\
\hline Mulata & 40 & 22,3 & 31 & 19,1 & 71 & 20,8 & & & \\
\hline Indígena & 1 & 0,6 & 0 & 0,0 & 1 & 0,3 & & & \\
\hline Amarela & 2 & 1,1 & 1 & 0,6 & 3 & 0,9 & & & \\
\hline Total & 179 & 100 & 162 & 100 & 341 & 100 & & & \\
\hline \multicolumn{10}{|l|}{ Medicamentos } \\
\hline Anti-hipertensivos & & & & & & & $8,861^{*}$ & 1 & $0,003^{\star \star \star}$ \\
\hline Usa & 75 & 41,9 & 43 & 26,5 & 118 & 34,6 & & & \\
\hline Não usa & 104 & 58,1 & 119 & 73,5 & 223 & 65,4 & & & \\
\hline \multicolumn{10}{|l|}{ Ansiolíticos } \\
\hline Usa & 164 & 91,6 & 125 & 77,2 & 289 & 84,8 & $13,757^{\star}$ & 1 & $0,000^{\star \star \star}$ \\
\hline Não usa & 15 & 8,4 & 37 & 22,8 & 53 & 15,2 & & & \\
\hline \multicolumn{10}{|l|}{ Antidepressivos } \\
\hline Usa & 161 & 89,9 & 136 & 84,0 & 297 & 87,1 & $2,718^{*}$ & 1 & 0,099 \\
\hline Não usa & 18 & 10,1 & 26 & 16,0 & 44 & 12,9 & & & \\
\hline \multicolumn{10}{|l|}{ Analgésicos } \\
\hline Usa & 155 & 86,6 & 113 & 69,8 & 268 & 78,6 & $14,332^{*}$ & 1 & $0,000^{\star \star \star}$ \\
\hline Não usa & 24 & 13,4 & 49 & 30,2 & 73 & 21,4 & & & \\
\hline
\end{tabular}

* Qui-quadrado de Pearson. ** Teste Exato de Fisher. ${ }^{* \star \star}$ Significativo.

Tabela 2. Análises descritivas e comparativas das variáveis idade, escolaridade (em anos) e renda familiar segundo 0 autorrelato de dificuldade para dormir - São Carlos, 2014

\begin{tabular}{|c|c|c|c|c|c|}
\hline \multirow{2}{*}{$\begin{array}{l}\text { Grupos } \\
\text { Variáveis }\end{array}$} & \multirow{2}{*}{$\begin{array}{c}\text { Sem dificuldade para } \\
\text { dormir }\end{array}$} & \multirow{2}{*}{$\begin{array}{c}\text { Com dificuldade para } \\
\text { dormir }\end{array}$} & \multirow{2}{*}{ Todos os grupos } & \multicolumn{2}{|c|}{ Análise comparativa } \\
\hline & & & & $\mathbf{U}^{\star}$ & $\mathbf{p}$ \\
\hline Idade (anos) & & & & 13365,50 & 0,212 \\
\hline Média & 69,33 & 69,93 & 69,61 & & \\
\hline Mediana & 67,00 & 69,00 & 68,00 & & \\
\hline Desvio-padrão & 7,30 & 6,91 & 7,12 & & \\
\hline Mín-Máx & $60-95$ & $60-98$ & $60-98$ & & \\
\hline Escolaridade (anos) & & & & 13401,00 & 0,217 \\
\hline Média & 4,06 & 3,54 & 3,81 & & \\
\hline Mediana & 4,00 & 3,00 & 4,00 & & \\
\hline Desvio-padrão & 3,77 & 3,23 & 3,52 & & \\
\hline Mín-Máx & $0,00-19,00$ & $0,00-15,00$ & $0,00-19,00$ & & \\
\hline Renda familiar (reais) & & & & 11832,00 & 0,427 \\
\hline Média & 2353,99 & 2249,79 & 2303,21 & & \\
\hline Mediana & 1800,00 & 1836,00 & 1810,00 & & \\
\hline Desvio-padrão & 1536,13 & 1621,70 & 1576,76 & & \\
\hline Mín-Máx & $724,00-10000,00$ & $724,00-10000,00$ & $724,00-10000,00$ & & \\
\hline
\end{tabular}

* Mann-Whitney. 
Nas tabelas 1 e 2, podemos observar que apenas as variáveis "sexo", "religião" e "uso de medicamentos" apresentaram diferenças significativas entre os grupos. Foram encontrados maior proporção de indivíduos do sexo feminino, menor número de católicos e maior número de evangélicos e espíritas e maior proporção de idosos que não usavam anti-hipertensivos, ansiolíticos e analgésicos no grupo com dificuldade de dormir. As análises comparativas evidenciaram não existir diferenças significativas entre os grupos em relação às demais variáveis sociodemográficas. Esse controle minimiza a possibilidade de varáveis intervenientes acarretarem vieses nos resultados.

\section{Análises descritivas e comparativas de estresse e sintomas depressivos em idosos segundo autorrelato de dificuldade para dormir}

Os escores referentes à Escala de Estresse Percebido são apresentados na tabela 3. O grupo sem dificuldade para dormir teve uma média de 15,97 pontos ( $M d=15,00, D P=$ $8,63, X_{\text {min }}=0,00, X_{\text {máx }}=46,00$ ) e a do grupo com dificuldade para dormir foi de 21,29 pontos ( $M d=20,00, D P=10,58$, $\left.X_{\text {min }}=0,00, X_{\text {máx }}=56,00\right)$. Essa diferença foi significativa $(U=$ 10034,50; $p=0,010$ ), portanto o nível de estresse do grupo de idosos com dificuldade para dormir é mais elevado que no grupo sem dificuldade para dormir (Figura 1, Tabela 3).
Além disso, os idosos com dificuldade para dormir tinham escores significativamente mais elevados nos itens $1,3,7,8,9,11$ e 13 da escala ( $p<0,05)$, evidenciando que eles eram indivíduos mais tristes, nervosos, irritados e com percepção negativa de afazeres, controle e gasto de tempo (Tabela 3).

Os dados relacionados aos sintomas depressivos são apresentados na tabela 4. Vale observar que o escore total do grupo sem dificuldade para dormir foi de 3,09 pontos (Md $=2,00, \mathrm{DP}=2,52, \mathrm{X}_{\text {min }}=0,00, \mathrm{X}_{\text {máx }}=13,00$ ) e o do grupo com dificuldade para dormir foi de 4,41 pontos ( $\mathrm{Md}=4,00, \mathrm{DP}=$ $\left.2,92, X_{\text {min }}=0,00, X_{\text {máx }}=13,00\right)$. Essa diferença foi significativa $(U=10427,50 ; p=0,000)$, portanto pode-se afirmar que $\mathrm{o}$ grupo de idosos com dificuldade para dormir apresentava maior número de sintomas depressivos (Figura 1, Tabela 4).

$\mathrm{Na}$ análise por item, os idosos com dificuldade para dormir tinham escores significativamente mais elevados nas questões 1, 2, 4, 11, 13, 14 e 15 da Escala de Depressão Geriátrica $(p<0,05)$, o que demonstra que os idosos sem dificuldade para dormir são mais satisfeitos com a vida, têm interesses e atividades, se aborrecem menos, valorizam a vida, se sentem com energia, têm esperança e se comparam positivamente em relação às outras pessoas (Tabela 4).

Tabela 3. Análises descritivas e comparativas de estresse segundo autorrelato de dificuldade para dormir - São Carlos, 2014

\begin{tabular}{|c|c|c|c|c|c|c|}
\hline \multirow{2}{*}{$\begin{array}{l}\text { Grupos } \\
\text { Itens de estresse }\end{array}$} & & \multirow[t]{2}{*}{$\begin{array}{c}\text { Sem } \\
\text { dificuldade } \\
\text { para } \\
\text { dormir }\end{array}$} & \multirow[t]{2}{*}{$\begin{array}{c}\text { Com } \\
\text { dificuldade } \\
\text { para } \\
\text { dormir }\end{array}$} & \multirow[t]{2}{*}{$\begin{array}{l}\text { Todos os } \\
\text { grupos }\end{array}$} & \multicolumn{2}{|c|}{ Análise comparativa } \\
\hline & & & & & $U^{*}$ & $p^{\star}$ \\
\hline \multirow[t]{3}{*}{ 1. Você tem ficado triste por causa de algo que aconteceu inesperadamente? } & Média & 1,82 & 2,48 & 2,13 & 11053,00 & $0,000^{*}$ \\
\hline & Mediana & 2,00 & 2,00 & 2,00 & & \\
\hline & $\mathrm{DP}$ & 1,53 & 1,47 & 1,53 & & \\
\hline \multirow{3}{*}{$\begin{array}{l}\text { 2. Você tem se sentido incapaz de controlar as coisas importantes em sua } \\
\text { vida? }\end{array}$} & Média & 0,93 & 1,09 & 1,01 & 13714,00 & 0,324 \\
\hline & Mediana & 0,00 & 0,00 & 0,00 & & \\
\hline & $\mathrm{DP}$ & 1,41 & 1,50 & 1,45 & & \\
\hline \multirow[t]{3}{*}{ 3. Você tem se sentido nervoso e "estressado"? } & Média & 1,59 & 2,20 & 1,88 & 10959,00 & $0,000^{*}$ \\
\hline & Mediana & 2,00 & 2,00 & 2,00 & & \\
\hline & $\mathrm{DP}$ & 1,29 & 1,43 & 1,39 & & \\
\hline \multirow[t]{3}{*}{ 4. Você tem tratado com sucesso dos problemas difíceis da vida? } & Média & 0,65 & 0,83 & 0,74 & 13306,50 & 0,138 \\
\hline & Mediana & 0,00 & 0,00 & 0,00 & & \\
\hline & $\mathrm{DP}$ & 1,02 & 1,12 & 1,07 & & \\
\hline \multirow{3}{*}{$\begin{array}{l}\text { 5. Você tem sentido que está lidando bem as mudanças importantes que } \\
\text { estão ocorrendo em sua vida? }\end{array}$} & Média & 0,58 & 0,82 & 0,70 & 13227,50 & 0,111 \\
\hline & Mediana & 0,00 & 0,00 & 0,00 & & \\
\hline & $\mathrm{DP}$ & 0,90 & 1,16 & 1,04 & & \\
\hline \multirow{3}{*}{$\begin{array}{l}\text { 6. Você tem se sentido confiante na sua habilidade de resolver problemas } \\
\text { pessoais? }\end{array}$} & Média & 0,46 & 0,69 & 0,57 & 13214,50 & 0,087 \\
\hline & Mediana & 0,00 & 0,00 & 0,00 & & \\
\hline & $\mathrm{DP}$ & 0,84 & 1,08 & 0,97 & & \\
\hline
\end{tabular}




\begin{tabular}{|c|c|c|c|c|c|c|}
\hline \multirow{2}{*}{$\begin{array}{l}\text { Grupos } \\
\text { Itens de estresse }\end{array}$} & & \multirow[t]{2}{*}{$\begin{array}{c}\text { Sem } \\
\text { dificuldade } \\
\text { para } \\
\text { dormir }\end{array}$} & \multirow[t]{2}{*}{$\begin{array}{c}\text { Com } \\
\text { dificuldade } \\
\text { para } \\
\text { dormir }\end{array}$} & \multirow[t]{2}{*}{$\begin{array}{l}\text { Todos os } \\
\text { grupos }\end{array}$} & \multicolumn{2}{|c|}{ Análise comparativa } \\
\hline & & & & & $\mathbf{U}^{*}$ & $\mathbf{p}^{*}$ \\
\hline \multirow{3}{*}{$\begin{array}{l}\text { 7. Você tem sentido que as coisas estão acontecendo de acordo com a sua } \\
\text { vontade? }\end{array}$} & Média & 1,48 & 1,85 & 1,66 & 12104,50 & $0,007^{*}$ \\
\hline & Mediana & 1,00 & 2,00 & 2,00 & & \\
\hline & DP & 1,38 & 1,32 & 1,36 & & \\
\hline \multirow{3}{*}{$\begin{array}{l}\text { 8. Você tem achado que não conseguiria lidar com todas as coisas que você } \\
\text { tem que fazer? }\end{array}$} & Média & 1,31 & 1,67 & 1,48 & 12422,00 & $0,018^{*}$ \\
\hline & Mediana & 1,00 & 2,00 & 1,00 & & \\
\hline & $\mathrm{DP}$ & 1,33 & 1,40 & 1,38 & & \\
\hline \multirow[t]{3}{*}{ 9. Você tem conseguido controlar as irritações em sua vida? } & Média & 0,61 & 1,08 & 0,83 & 11223,50 & $0,000^{*}$ \\
\hline & Mediana & 0,00 & 1,00 & 0,00 & & \\
\hline & DP & 1,00 & 1,24 & 1,15 & & \\
\hline \multirow[t]{3}{*}{ 10. Você tem sentido que as coisas estão sob o seu controle? } & Média & 0,81 & 1,06 & 0,93 & 12987,50 & 0,069 \\
\hline & Mediana & 0,00 & 1,00 & 0,00 & & \\
\hline & DP & 1,11 & 1,26 & 1,19 & & \\
\hline \multirow{3}{*}{$\begin{array}{l}\text { 11. Você tem ficado irritado porque as coisas que acontecem estão fora do } \\
\text { seu controle? }\end{array}$} & Média & 1,85 & 2,42 & 2,12 & 11424,50 & $0,000^{*}$ \\
\hline & Mediana & 2,00 & 2,00 & 2,00 & & \\
\hline & DP & 1,49 & 1,44 & 1,49 & & \\
\hline \multirow[t]{3}{*}{ 12. Você tem se encontrado pensando sobre as coisas que deve fazer? } & Média & 2,56 & 2,89 & 2,72 & 13057,00 & 0,086 \\
\hline & Mediana & 3,00 & 4,00 & 4,00 & & \\
\hline & $\mathrm{DP}$ & 1,62 & 1,45 & 1,55 & & \\
\hline \multirow[t]{3}{*}{ 13. Você tem conseguido controlar a maneira como gasta seu tempo? } & Média & 0,44 & 0,75 & 0,59 & 12708,50 & $0,014^{*}$ \\
\hline & Mediana & 0,00 & 0,00 & 0,00 & & \\
\hline & DP & 0,91 & 1,20 & 1,06 & & \\
\hline \multirow{3}{*}{$\begin{array}{l}\text { 14. Você tem sentido que as dificuldades se acumulam a ponto de você } \\
\text { acreditar que não pode superá-las? }\end{array}$} & Média & 0,87 & 1,47 & 1,16 & 11384,00 & $0,000^{*}$ \\
\hline & Mediana & 0,00 & 1,00 & 0,00 & & \\
\hline & $\mathrm{DP}$ & 1,24 & 1,53 & 1,41 & & \\
\hline \multirow[t]{3}{*}{ TOTAL DE ESTRESSE } & Média & 15,97 & 21,29 & 18,50 & 10034,50 & $0,000^{*}$ \\
\hline & Mediana & 15,00 & 20,00 & 17,00 & & \\
\hline & $\mathrm{DP}$ & 8,63 & 10,58 & 9,95 & & \\
\hline
\end{tabular}

${ }^{*}$ Mann-Whitney. ${ }^{\star *}$ Significativo.

Tabela 4. Análises descritivas e comparativas de depressão segundo autorrelato de dificuldade para dormir e análise comparativa entre os grupos - São Carlos, 2014

\begin{tabular}{|c|c|c|c|c|c|c|}
\hline Grupos & & $\begin{array}{c}\text { Sem } \\
\text { dificuldade } \\
\text { para } \\
\text { dormir }\end{array}$ & $\begin{array}{c}\text { Com } \\
\text { dificuldade } \\
\text { para } \\
\text { dormir }\end{array}$ & $\begin{array}{l}\text { Todos os } \\
\text { grupos }\end{array}$ & \multicolumn{2}{|c|}{ Análise comparativa } \\
\hline Itens de depressão & & & & & $\mathbf{U}^{*}$ & $\mathbf{p}$ \\
\hline \multirow[t]{3}{*}{ 1. Você está satisfeito com sua vida? } & Média & 0,06 & 0,14 & 0,10 & 13250,50 & $0,007^{\star \star}$ \\
\hline & Mediana & 0,00 & 0,00 & 0,00 & & \\
\hline & DP & 0,23 & 0,35 & 0,30 & & \\
\hline \multirow[t]{3}{*}{ 2. Você deixou muitos dos seus interesses e atividades? } & Média & 0,47 & 0,61 & 0,54 & 12523,50 & $0,012^{\star *}$ \\
\hline & Mediana & 0,00 & 1,00 & 1,00 & & \\
\hline & $\mathrm{DP}$ & 0,50 & 0,49 & 0,50 & & \\
\hline
\end{tabular}




\begin{tabular}{|c|c|c|c|c|c|c|}
\hline Grupos & & $\begin{array}{c}\text { Sem } \\
\text { dificuldade }\end{array}$ & $\begin{array}{c}\text { Com } \\
\text { dificuldade }\end{array}$ & Todos os & Análise $c$ & parativa \\
\hline Itens de depressão & & & & & $\mathbf{U}^{*}$ & $\mathbf{p}$ \\
\hline 3. Você sente que sua vida está vazia? & Média & 0,21 & 0,30 & 0,26 & 13191,50 & 0,057 \\
\hline & Mediana & 0,00 & 0,00 & 0,00 & & \\
\hline & DP & 0,41 & 0,46 & 0,44 & & \\
\hline 4. Você se aborrece com frequência? & Média & 0,26 & 0,47 & 036 & 11504,00 & $0,000^{\star \star}$ \\
\hline & Mediana & 0,00 & 0,00 & 0,00 & & \\
\hline & DP & 0,44 & 0,50 & 0,48 & & \\
\hline 5. Você se sente de bom humor a maior parte do tempo? & Média & 0,10 & 0,17 & 0,13 & 13451,00 & 0,051 \\
\hline & Mediana & 0,00 & 0,00 & 0,00 & & \\
\hline & DP & 0,30 & 0,38 & 0,34 & & \\
\hline 6. Você tem medo de que algum mal vá Ihe acontecer? & Média & 0,39 & 0,48 & 0,43 & 13277,50 & 0,117 \\
\hline & Mediana & 0,00 & 0,00 & 0,00 & & \\
\hline & DP & 0,49 & 0,50 & 0,50 & & \\
\hline 7. Você se sente feliz a maior parte do tempo? & Média & 0,09 & 0,12 & 0,11 & 14005,00 & 0,307 \\
\hline & Mediana & 0,00 & 0,00 & 0,00 & & \\
\hline & DP & 0,29 & 0,33 & 0,31 & & \\
\hline 8. Você sente que sua situação não tem saída? & Média & 0,13 & 0,17 & 0,15 & 13937,00 & 0,321 \\
\hline & Mediana & 0,00 & 0,00 & 0,00 & & \\
\hline & DP & 0,34 & 0,38 & 0,36 & & \\
\hline 9. Você prefere ficar em casa ao invés de sair e fazer coisas novas? & Média & 0,51 & 0,55 & 0,53 & 13904,50 & 0,449 \\
\hline & Mediana & 1,00 & 1,00 & 1,00 & & \\
\hline & DP & 0,50 & 0,50 & 0,50 & & \\
\hline 10. Você se sente com mais problemas de memória do que a maioria? & Média & 0,22 & 0,30 & 0,26 & 13353,50 & 0,098 \\
\hline & Mediana & 0,00 & 0,00 & 0,00 & & \\
\hline & DP & 0,42 & 0,46 & 0,44 & & \\
\hline 11. Você acha maravilhoso estar vivo? & Média & 0,01 & 0,06 & 0,03 & 13685,00 & $0,003^{\text {** }}$ \\
\hline & Mediana & 0,00 & 0,00 & 0,00 & & \\
\hline & DP & 0,08 & 0,24 & 0,18 & & \\
\hline 12. Você se sente um inútil nas atuais circunstâncias? & Média & 0,13 & 0,19 & 0,16 & 13758,00 & 0,197 \\
\hline & Mediana & 0,00 & 0,00 & 0,00 & & \\
\hline & DP & 0,34 & 0,39 & 0,37 & & \\
\hline 13. Você se sente cheio de energia? & Média & 0,16 & 0,28 & 0,22 & 12731,00 & $0,007^{\star \star}$ \\
\hline & Mediana & 0,00 & 0,00 & 0,00 & & \\
\hline & DP & 0,37 & 0,45 & 0,42 & & \\
\hline 14. Você acha que sua situação é sem esperanças? & Média & 0,09 & 0,18 & 0,13 & 13199,50 & $0,015^{\star \star}$ \\
\hline & Mediana & 0,00 & 0,00 & 0,00 & & \\
\hline & DP & 0,29 & 0,39 & 0,34 & & \\
\hline 15. Você sente que a maioria das pessoas está melhor que você? & Média & 0,25 & 0,38 & 0,31 & 12514,00 & $0,006^{\star \star}$ \\
\hline & Mediana & 0,00 & 0,00 & 0,00 & & \\
\hline & DP & 0,43 & 0,49 & 0,46 & & \\
\hline TOTAL DE DEPRESSÃOO & Média & 3,09 & 4,41 & 3,72 & 10427,50 & $0,000^{* *}$ \\
\hline & Mediana & 2,00 & 4,00 & 3,00 & & \\
\hline & DP & 2,52 & 2,92 & 2,80 & & \\
\hline
\end{tabular}

* Mann-Whitney. ${ }^{* \star}$ Significativo. 

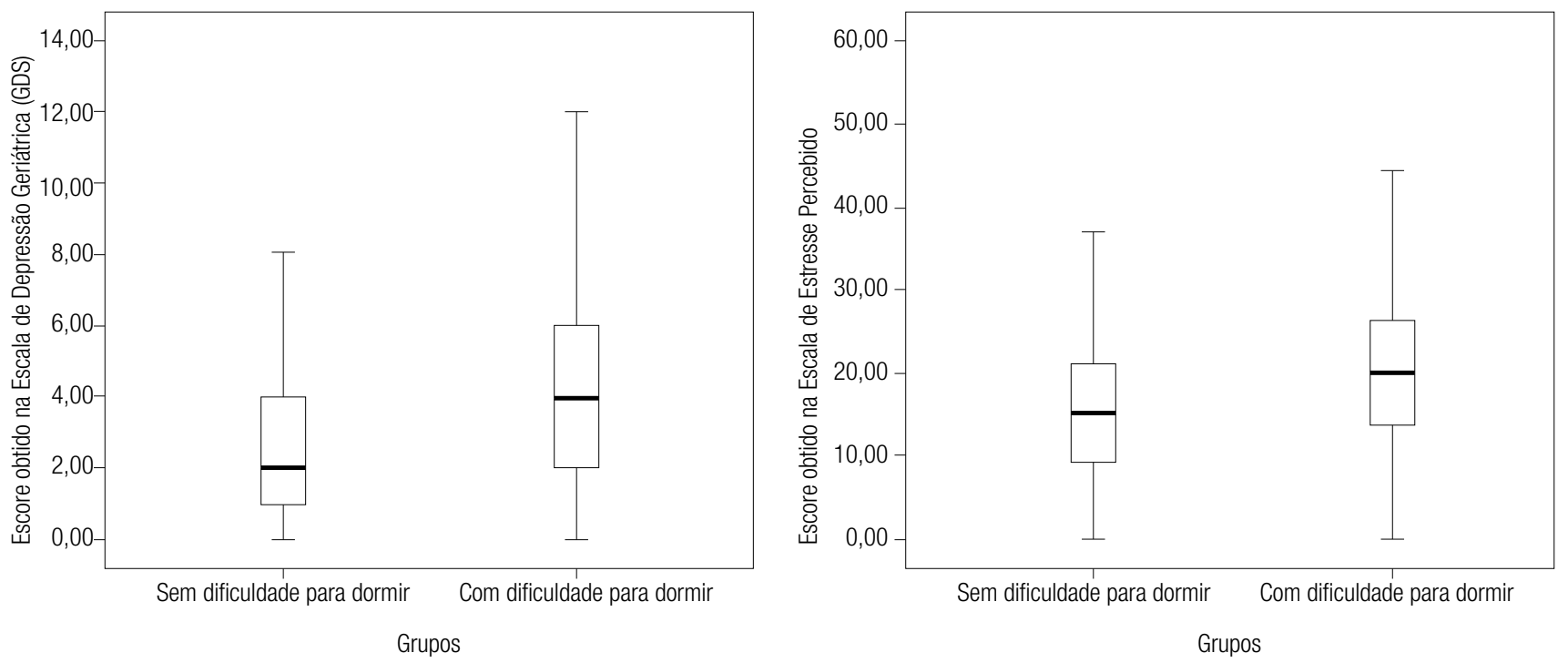

Figura 1. Comparativo dos escores na escala de estresse percebido dos idosos sem e com dificuldade para dormir e comparativo dos Escores na Escala de Depressão Geriátrica os idosos sem e com dificuldade para dormir - São Carlos, 2014.

\section{DISCUSSÃO}

Esse estudo teve como objetivo identificar associação entre variáveis emocionais (estresse e sintomas depressivos) e o autorrelato de dificuldade para dormir em idosos. Os resultados obtidos identificaram que os idosos com dificuldade para dormir são indivíduos mais tristes, nervosos, irritados e com percepção negativa de afazeres, controle e gasto de tempo, e as mulheres apresentam maior dificuldade para dormir que os homens.

O nível de estresse do grupo de idosos com dificuldade para dormir é mais elevado que no grupo sem dificuldade para dormir, assim como eles também apresentavam maior número de sintomas depressivos.

A predominância de idade inferior a 75 anos pode ser explicada pela expectativa de vida do brasileiro que na época da coleta de dados, 2014, era de 75,2 anos ${ }^{27}$. Dessa forma, é esperado que os usuários da atenção básica sejam mais jovens. Os idosos mais longevos vão necessitar de serviços especializados de saúde ${ }^{28}$.

Podemos observar também uma feminilização na amostra, o que acarreta a baixa escolaridade apresentada. Isso porque os modelos de família tradicionais dos anos 1970 colocavam o homem como provedor e a mulher como cuidadora dos filhos e do lar. Sendo assim, não se esperava alto nível de instrução das mulheres, mas habilidades de cuidado e destreza para os afazeres domésticos ${ }^{29}$.

Vale destacar um percentual elevado de idosos que apresentam dificuldade para dormir autorrelatada (47,5\%). 0 número relativo de mulheres que apresentaram dificuldade para dormir foi maior (83,3\%). Dados semelhantes publicados por Moreno et al..$^{29}$ apontaram que mais de $50 \%$ dos indivíduos com idade superior a 65 anos apresentam queixas relacionadas ao sono, com maior prevalência sobre o sexo feminino. Os estudos de Oliveira et al. ${ }^{30}$ e de Zanuto et al. ${ }^{31}$ também indicam que as mulheres estão mais propensas a ter sintomas depressivos e estressores relacionados a conflitos familiares que podem causar distúrbios do sono.

Uma pobre qualidade de sono pode se associar a queixas de humor, como, por exemplo, fadiga, ansiedade, irritabilidade e humor depressivo. Raposo e Veríssimo ${ }^{32}$ apresentam a relação entre a dificuldade para dormir e os sintomas depressivos com evidências de que a insônia matinal prediz o aparecimento dos sintomas depressivos. A hipótese que sustenta a relação entre qualidade do sono e sintomas depressivos é a resposta positiva contra a insônia quando há a administração de antidepressivos, e os sintomas depressivos quando a qualidade de sono é melhorada.

Além disso, Quinhones e Gomes ${ }^{33}$ afirmam que os sintomas depressivos e transtornos de ansiedade são frequentes entre pessoas com mais de 65 anos de idade, por consequência de perda do cônjuge, aposentadoria, isolamento social, doenças e declínio cognitivo, que são fatores de risco para sintomas depressivos em idosos. A depressão e os transtornos de ansiedade, por sua vez, contribuem para a insônia e outros distúrbios do sono.

No que se refere aos níveis de estresse, ansiedade e sintomas depressivos em relação à qualidade de sono, Galvão et al..$^{34}$ demonstram que existe uma relação significativa entre eles. Perturbações do sono influenciam negativamente a qualidade de vida, gerando situações de estresse, irritabilidade, impaciência e indisponibilidade, agressividade, distrações e apatia, bem como situações de hiperatividade, desconforto, dificuldade de interação, tristeza, isolamento e falta de ânimo e de energia. 
Um estudo realizado em uma população idosa na Ásia ( $n=107)$ com o objetivo de analisar a associação entre sono e sintomas psiquiátricos investigou qualidade do sono, sintomas depressivos e ansiedade. Os resultados permitiram observar que os sintomas depressivos e a ansiedade apresentaram-se significativamente correlacionados com problemas de sono $(p<0,001)^{35}$.

Outro estudo com 3.230 idosos, realizado em Xiamen, na China, permitiu observar que quanto melhor a qualidade do sono dos idosos ( $B=0,04, p<0,01$ ), menos sintomas depressivos eram apresentados $(B=-0,40, p<0,001)^{36}$.

No que tange ao estresse, uma pesquisa de saúde comunitária teve como objetivo explorar a correlação entre a duração do sono e o estresse percebido em uma amostra de 133.44 coreanos em idade adulta. Os resultados desse estudo permitiram concluir que o estresse apresentava maiores escores quando os indivíduos dormiam por menos horas. Contudo, os dados mostraram que quanto mais velhos, os participantes apresentavam-se mais estressados ${ }^{37}$.

Quanto às limitações, esta pesquisa não permitiu fazer generalizações. Embora o cálculo amostral tenha indicado que o número de idosos era capaz de representar poder estatístico, tratou-se de uma amostra única de idosos atendidos em USFs de uma cidade do interior paulista. Ademais, confundidores como tipos de medicamentos em uso não foram potencialmente explorados a fim de garantir a minimização de vieses. Estudos futuros com amostras e análises robustas que possam garantir tais extrapolações são recomendados. Com base nos dados encontrados, pode-se ter uma visão mais específica de como os sintomas depressivos e o estresse interferem na boa qualidade de sono de idosos atendidos na atenção básica. O reconhecimento e o tratamento precoce são indispensáveis para o cuidado integral. Este estudo faz parte do esforço de desmistificar que problemas de sono e distúrbios emocionais fazem parte do envelhecimento.

Ademais, a prevalência de sintomas depressivos e queixas relacionadas ao sono em idosos são temas estudados em contextos muito diversos, ambulatórios, hospitais, clínicas especializados. Porém, na atenção básica a prevalência é alta. Estudos que possam subsidiar as estratégias e políticas de saúde em prol da pessoa idosa são imprescindíveis. Desse modo, este estudo faz parte do esforço de evidenciar a importância do diagnóstico e do tratamento de indivíduos que sofrem de sintomas depressivos e distúrbios do sono em todos os níveis de atenção.

\section{CONCLUSÃO}

Podemos identificar que há associação entre variáveis emocionais (estresse e sintomas depressivos) e o autorrelato de dificuldade para dormir em idosos. Foi observado que idosos com dificuldade para dormir são indivíduos mais tristes, nervosos, irritados e com percepção negativa de afazeres, controle e gasto de tempo. Mulheres também apresentaram maior dificuldade para dormir se comparadas aos homens. Conclui-se que o nível de estresse e a quantidade de sintomas depressivos do grupo de idosos com dificuldade para dormir são mais elevados que no grupo sem dificuldade para dormir.

Cabe ressaltar a importância dos dados obtidos neste trabalho, não só para futuras pesquisas, mas também para que haja a intervenção profissional precoce e para subsidiar estratégias e políticas públicas que visem ao trabalho terapêutico com idosos, estimulando a reflexão sobre condutas positivas de enfrentamento e sobre comportamentos resilientes em relação a situações adversas.

\section{CONTRIBUIÇÕES INDIVIDUAIS}

Grazielle Ferreira Iroldi, Bruna Moretti Luchesi, Sofia Cristina lost Pavarini e Keika Inouye - Contribuíram na concepção e no desenho do estudo.

Grazielle Ferreira Iroldi e Keika Inouye - Participaram da elaboração do artigo e da análise e interpretação de dados.

Élen dos Santos Alves, Juliana de Fátima Zacarin Cardoso e Sofia Cristina lost Pavarini - Participaram da revisão crítica relevante do conteúdo intelectual.

Todos os autores participaram da aprovação final da versão a ser publicada e são responsáveis por todos os aspectos do trabalho, na garantia da exatidão e integridade de qualquer parte da obra.

\section{FINANCIAMENTO}

O estudo recebeu apoio financeiro da Coordenação de Aperfeiçoamento de Pessoal de Nível Superior (Capes), processo no 304067/2015-6.

\section{CONFLITO DE INTERESSES}

Os autores declaram não haver conflito de interesses.

\section{AGRADECIMENTOS}

Agradeço a todos que participaram deste estudo e ao Capes, pelo apoio financeiro recebido.

\section{REFERÊNCIAS}

1. Dantas CMHL, Bello FA, Barreto KL, Lima LS. Capacidade funcional de idosos com doenças crônicas residentes em Instituições de Longa Permanência. Rev Bras Enferm. 2013;66(6):914-20. 
2. Silva AA, Mello RGB, Schaan CW, Fuchs FD, Redline S, Fuchs SC. Sleep duration and mortality in the elderly: a systematic review with meta-analysis. BMJ Open. 2016;6(2):1-13.

3. Geib LTC, Cataldo Neto A, Wainberg R, Nunes ML. Sono e envelhecimento. Rev Psiquiatr Rio Gd Sul. 2003;25(3):453-65.

4. Téllez A, Juárez-García DM, Jaime-Bernal L, García-Cadena C. Prevalencia de trastornos de sueño en relación con factores sociodemográficos y depresión en adultos mayores de Monterrey, México. Rev Colomb Psicol. 2016;25(1):95-106

5. Mugeiro MJC. Qualidade do Sono nos Idosos [Internet]. Instituto Politécnico de Viseu, Escola Superior de Saúde de Viseu; 2011. Disponível em: http://hdl.handle.net/10400.19/1658. Acesso em: 20 mar. 2020.

6. Müller MR, Guimarães SS. Impacto dos transtornos do sono sobre o funcionamento diário e a qualidade de vida. Estud Psicol. 2007;24(4):519-28.

7. Lucchesi LM, Pradella-Hallinan M, Lucchesi M, Moraes WAS. 0 sono em transtornos psiquiátricos. Rev Bras Psiquiatr. 2005;27(Suppl 1):27-32.

8. Ferreira ODL. A relação entre transtorno da insônia e funções executivas em adultos [dissertação]. Natal: Universidade Federal do Rio Grande do Norte, Centro de Ciências Humanas, Letras e Artes; 2014.

9. Lorenzini LM, Coelho AT, Rossini S, Proença C, Oliveira JC, Rovere HD, et al. Sono e atividade física: considerações atuais. In: Reimão R (Org.). Distúrbios do sono. 1a ed. São Paulo: Associação Paulista de Medicina; 2003.

10. American Psychiatric Association. Diagnostic and statistical manual of mental disorders. 5th ed. Washington, DC: Press, American Psychiatric; 2013.

11. Vilela LHM, Juruena MF. Avaliação do funcionamento do eixo HPA em deprimidos por meio de medidas basais: revisão sistemática da literatura e análise das metodologias utilizadas. J Bras Psiquiatr. 2014;63(3):232-41.

12. Ferreira MB, Ceolim MF, Cupertino APFB, Fortes-Burgos ACG. Eventos estressantes associados ao cuidado, a alterações de sono e a sintomas depressivos entre idosos residentes na comunidade. Geriatr Gerontol Aging. 2009;3(2):95-102.

13. Hoffmann EJ, Ribeiro F, Farnese JM, Lima EWB. Sintomas depressivos e fatores associados entre idosos residentes em uma comunidade no norte de Minas Gerais, Brasil. J Bras Psiquiatr. 2010;59(3):190-7.

14. Cole MG, Dendukuri N. Risk factors for depression among elderly community subjects: A systematic review and meta-analysis. Am J Psychiatry. 2003;160(6):1147-56.

15. Menezes-Silva R, Oliveira DWD, Biscaro PCB, Orti NP, Sá-Pinto AC, Ramos-Jorge ML. Inquérito epidemiológico em população idosa (parte II): saúde bucal, ansiedade, depressão, estresse e uso de medicamentos. Sci Med (Porto Alegre). 2016;26(1):1-8.

16. Xavier FM, Ferraz MP, Trenti CM, Argimon I, Bertolucci PH, Poyares D, et al. Transtorno de ansiedade generalizada em idosos com oitenta anos ou mais. Rev Saúde Pública. 2001;35(3):294-302.

17. Sociedade Brasileira de Sono. I Consenso Brasileiro sobre Insônia. Hypnos. 2003;(4).

18. Chellappa SL, Araújo JF. 0 sono e os transtornos do sono na depressão. Rev Psiquiatr Clin. 2007;34(6):285-9.

19. Fundação Seade. População: São Carlos, 2010. Disponível em: http://produtos.seade.gov. $\mathrm{br} /$ produtos/retratosdesp/view/index.php?porTema=true\&locld=3548906\&temald=1\& pag=1. Acesso em: 2 jul. 2020.

20. Fundação Seade. Perfil dos municípios paulistas: São Carlos, 2020. Disponível em: https:// perfil.seade.gov.br. Acesso em: 2 jul. 2020.
21. Prefeitura Municipal de São Carlos. Programa Saúde da Família - PSF, 2020. Disponível em: http://www.saocarlos.sp.gov.br/index.php/saude/115417-programa-saude-da-familiapsf.html. Acesso em: 2 jul. 2020.

22. Brasil. Ministério da Saúde. Conselho Nacional de Saúde. Resolução nº 466, de 12 de dezembro de 2012. Aprova diretrizes e normas regulamentadoras de pesquisas envolvendo seres humanos. Brasília, Diário Oficial da União, 12 dez. 2012.

23. Cohen S, Kamarck T, Mermelstein R. A global measure of perceived stress. J Health Soc Behavior. 1983;24:385-96.

24. Luft, CB, Sanches S0, Mazo GZ, Andrade A. Versão brasileira da Escala de Estresse Percebido: tradução e validação para idosos. Rev Saúde Pública. 2007;41(4):606-15.

25. Yesavage JA, Brink TL, Rose TL, Lum O, Huang V, Adey M, et al. Development and validation of a geriatric depression screening scale: a preliminary report. J. Psychiatr. Res. 1983;17(1):37-49.

26. Almeida OP, Almeida SA. Short Versions of the Geriatric Depression Scale: a study of their validity for the diagnosis of a major depressive episode according to ICD-10 and DSM-IV. Int J Geriatric Psych. 1999;14:858-65.

27. Instituto Brasileiro de Geografia e Estatística. Tábua completa de mortalidade para 0 Brasil-2014: breve análise da evolução da mortalidade no Brasil. Rio de Janeiro; 2015.

28. Soares C. A distribuição do tempo dedicado aos afazeres domésticos entre homens $e$ mulheres no âmbito da família. XVI Encontro Nacional de Estudos Populacionais. Caxambú - MG; 2008. p. 1-19.

29. Moreno CRC, Santos JLF, Lebrão ML, Ulhôa MA, Duarte YA0. Sleep disturbances in older adults are associated to female sex, pain and urinary incontinence. Rev Bras Epidemiol. 2018;21(Suppl 2).

30. Oliveira MF, Bezerra VP, Silva A0, Alves MS, Moreira MSP, et al. The symptomatology of self-referred depression by elderly people who live in a shantytown. Ciênc Saúde Coletiva. 2012;17(8):2191-8.

31. Zanuto ACE, Lima CSM, Araújo GR, Silva PE, Anzolin CC, Araujo YCM, et al. Distúrbios do sono em adultos de uma cidade do estado de São Paulo. Rev Bras Epidemiol. 2015;18(1):42-53.

32. Raposo FMO, Veríssimo MTM. As alterações do sono no idoso [dissertação]. Coimbra: Faculdade de Medicina da Universidade de Coimbra; 2015.

33. Quinhones MS, Gomes MM. Sono no envelhecimento normal e patológico: aspectos clínicos e fisiopatológicos. Rev Bras Neurol. 2011;47(1):31-42.

34. Galvão A, Pinheiro M, Gomes MJ, Ala S. Ansiedade, stress e depressão relacionados com perturbações do sono-vigília e consumo de álcool em alunos do ensino superior. Rev Port Enferm Saúde Ment. 2017;5(spe5):8-12.

35. Yu J, Rawtaer I, Fam J, Jiang MJ, Feng L, Kua EH, et al. Sleep correlates of depression and anxiety in an elderly Asian population. Psychogeriatrics. 2016;16(3):191-5.

36. Yuan M, Fu H, Liu R, Fang Y. Effect of frequency of exercise on cognitive function in older adults: Serial mediation of depression and quality of sleep. Int J Environ Res Public Health. 2020;17(3):709.

37. Kim HJ, Oh SY, Joo JH, Dong-Woo C, Eun-Cheol P. The relationship between sleep duration and perceived stress: Findings from the 2017 community health survey in Korea. Int J Environ Res Public Health. 2019;16(17):3208. 